$45 \mid 2010$

Langue française, identité(s) et école(s) : le cas de la minorité catholique au Levant (milieu XIX ${ }^{\mathrm{e}}-\mathrm{XX}^{\mathrm{e}}$

siècles). Représentations identitaires et apprentissage des langues : Europe, bassin méditerranéen ( $\left.\mathrm{XV}\right|^{\mathrm{e}}-\mathrm{XX}{ }^{\mathrm{e}}$ siècles)

\title{
Le rôle de l'hygiène dans le discours de la méthode directe en Bretagne et en Tornédalie, 1880-1925
}

Julia Nordblad

\section{OpenEdition}

Édition électronique

URL : https://journals.openedition.org/dhfles/2469

DOI : $10.4000 /$ dhfles.2469

ISSN : 2221-4038

Éditeur

Société Internationale pour l'Histoire du Français Langue Étrangère ou Seconde

Édition imprimée

Date de publication : 1 juin 2010

Pagination : 177-197

ISSN : 0992-7654

\section{Référence électronique}

Julia Nordblad, «Le rôle de l'hygiène dans le discours de la méthode directe en Bretagne et en Tornédalie, 1880-1925 », Documents pour l'histoire du français langue étrangère ou seconde [En ligne], 45 | 2010, mis en ligne le 10 février 2014, consulté le 27 mai 2021. URL : http://

journals.openedition.org/dhfles/2469; DOI : https://doi.org/10.4000/dhfles.2469

Ce document a été généré automatiquement le 27 mai 2021.

(c) SIHFLES 


\title{
Le rôle de l'hygiène dans le discours de la méthode directe en Bretagne et en Tornédalie, 1880-1925
}

\author{
Julia Nordblad
}

\section{Introduction}

\subsection{Nationalisme international}

1 Depuis deux siècles, la plupart des pays européens se sont engagés dans l'invention des identifications nationales (Anderson 1983; Brubaker et Cooper 2005: 71-73; Thiesse 1999) ${ }^{1}$. Comme le note Anne-Marie Thiesse, «rien de plus international que la formation des identités nationales. Le paradoxe est de taille puisque l'irréductible singularité de chaque identité nationale a été le prétexte d'affrontements sanglants » (Thiesse 2001a : 11). Ces efforts pour produire des communautés imaginaires furent non seulement modelés sur les mêmes principes et à peu près en même temps, mais ce processus s'est aussi déroulé en étant accompagné de vives discussions internationales, entre pédagogues, hommes et femmes, intellectuels, politiques, etc. Bref, le débat des différentes techniques nationalisantes était également international.

The common model for the generation of national identities was forged by European intellectuals in the course of the 19th century through a process of mutual observation, imitation and transfer of ideas and expertise. (Thiesse 1999)

\subsection{Questions}

Les politiques d'identification, quoique fondées sur le même modèle, différaient néanmoins selon les pays, par exemple dans l'importance donnée à la langue, à l'origine ou à l'histoire. Dans ce texte, nous examinerons le rôle d'un courant de pensée médical et politique, l'hygiénisme, dans une méthode pédagogique d'enseignement de la langue. Nous poserons la question de savoir comment cette influence fit converger les 
approches pédagogiques du problème de la différence linguistique dans la nation, en Suède et en France.

Nous prenons comme point de départ le fait que, à l'époque qui nous concerne, l'école a joué un rôle décisif aussi bien dans l'unification nationale et linguistique que dans la propagation d'un nouveau régime hygiénique (Csergo 1988: 107-110; Thiesse 2001a : 240). Nous examinerons de plus près l'introduction de la méthode directe dans l'enseignement de la langue nationale dans deux régions alloglottes, en France et en Suède: la Bretagne bretonnante en France, et la Tornédalie (Tornedalen) en Suède, région où la plupart des habitants parlaient une variante régionale du finnois, le meänkieli. Le but principal est de décrire et d'analyser le rôle joué par le courant croissant de l'hygiénisme dans les discours de la méthode directe, exemple d'une technique visant à unir le pays par la langue nationale. À travers cette analyse, nous cherchons à examiner la thèse de la similarité de la génération des identifications nationales, thèse avancée par Anne-Marie Thiesse.

\section{La France}

\subsection{Situation linguistique}

4 Vers la fin du XIX ${ }^{e}$ siècle, les différentes langues régionales étaient toujours dominantes en France. À la suite de la guerre de 1870-71, de nombreux intellectuels en France se tournèrent vers la question de l'école. Selon eux, la défaite face à l'armée prussienne s'expliquait par l'incapacité du système éducatif français à inculquer le sens du patriotisme aux enfants.

Ces préoccupations expliquent que la réorganisation de l'enseignement après 1870 est beaucoup plus qu'une question de programmes nouveaux, ou qu'un problème de politique intérieure : il s'agit véritablement d'une refonte de l'esprit national, imposée par le souvenir de la défaite. D'où l'aspect patriotique que prennent, pour l'opinion publique, les réformes projetées. (Digeon 1959 : 365-366)

6 La production d'une conscience nationale fut alors l'un des buts principaux des réformes du système scolaire dans les années 1880. (Gosnell $2002: 42$ )

Dans ce climat patriotique, la question de l'unité linguistique prit davantage d'importance. Vers 1880, à l'époque des lois Ferry, l'idée que l'école primaire doive être exclusivement francophone gagna de l'influence dans les cercles politiques : un rapport scolaire détaillé souligna la nécessité de franciser la Bretagne, et plusieurs pédagogues se tournèrent vers cette région. Au tournant du vingtième siècle, la balance commença à changer de sens en faveur de la langue française. (Chanet 1996: 206, 216; Weber $1976: 312)$

8 Le processus d'unification linguistique de la France, nommé "génocide culturel » par certains, n'est cependant pas achevé, même aujourd'hui : les idiomes locaux subsistent (Chanet 1996: 203). Plusieurs études historiques des dernières années soulignent l'importance des petites patries pour le nationalisme français (Thiesse 2001b: 79). L'inculcation d'une identification nationale ne voulait pas dire éradication de la culture régionale. La tendance à une attitude hostile aux patois était répandue dans la Troisième République mais elle n'était pas générale, et la pratique dans les salles de classe n'était pas toujours identique à celle qui était prescrite dans les documents 
officiels (Chervel 2006: 29; Ozouf 1996: 9-11; Thiesse 2001b: 79). Néanmoins, la tendance francisante était forte, surtout en Bretagne et, dans les années 1880 et 1890, au centre des débats, on trouve la méthode directe.

\subsection{La méthode directe}

9 Pour le dire brièvement, la méthode directe est une technique par laquelle on enseigne une langue dans cette langue même, sans l'intermédiaire d'une deuxième langue, habituellement la langue maternelle de l'élève. En Bretagne, les autorités choisirent la méthode directe comme outil principal dans l'enseignement primaire. Celui qui attacha son nom à ce changement pédagogique s'appelait Irénée Carré (1829-1909).

Carré, agrégé de grammaire, commença sa carrière en tant que professeur dans divers endroits en France, notamment au lycée de Rennes. Il devint inspecteur d'académie en 1870, et inspecteur général de l'enseignement primaire treize ans plus tard. Proche du directeur de l'enseignement primaire, Ferdinand Buisson, Carré était sans doute un personnage qui gravitait autour des décideurs pédagogiques de l'époque. Durant sa carrière, il avait été chargé d'inspecter des écoles bretonnes, ce qui avait inspiré son intérêt pour la pédagogie de la langue nationale. En 1886-87, il fut chargé d'une mission destinée à élaborer une méthode pédagogique pour aider l'agrégation de la Bretagne à la communauté nationale (Chanet 1996 : 217). Il s'engagea également dans la question de l'enseignement du français aux enfants alloglottes en général, et fit plusieurs tournées dans les régions concernées. (Puren $2004: 174$; Boutan 1998 : 50)

Pourtant, comme le note Puren, ce ne fut guère Carré qui inventa la méthode directe. Elle était en effet déjà en usage en Alsace pendant la première moitié du XIX ${ }^{\mathrm{e}}$ siècle. Charles Vivien, professeur à l'école normale de Strasbourg, qui avait fait ses études en Allemagne, fut probablement le premier à introduire la méthode en France (Puren $2003: 35)$. Néanmoins, Carré devint célèbre en tant que grand promoteur de la méthode à laquelle plusieurs pédagogues de l'époque faisaient référence sous le terme de méthode Carré. En 1889, l'inspecteur général partit pour une grande tournée dans les trois départements bretonnants, donnant des conférences pour lancer sa méthode. (Chanet $1996: 220$ )

\section{La Suède}

\subsection{Situation linguistique en Suède}

12 Comme en France, il y avait en Suède au XIX ${ }^{\mathrm{e}}$ siècle des régions où la plupart des habitants ne parlaient pas la langue nationale. Entre autres langues parlées en Suède on avait différents dialectes de same, le finnois et le meänkieli. Durant le XIX ${ }^{e}$ siècle, l'État suédois menait une politique de langues délibérée de suédisation (Elenius $2006: 152$ ). À partir des années 1870 , les programmes scolaires de l'école primaire consacraient davantage d'heures à la langue dite maternelle (c'est-à-dire le suédois) qu'à l'instruction religieuse, et la différence entre les deux continua de s'accroître jusqu'au tournant du siècle (Mellberg 1996: 52). Les autorités suédoises commencèrent à s'intéresser plus sérieusement aux régions alloglottes du nord du pays, et les finnophones de la Tornédalie devinrent une vraie question politique. La motivation en était l'inquiétude que les Tornédaliens ne se tournassent vers la Finlande, et il fallait 
donc les attacher à la Suède par l'éducation primaire. La langue d'éducation attira l'attention des éducateurs, hommes d'Église et hommes politiques. L'école devint au fur et à mesure exclusivement suédophone, le finnois disparut de l'éducation primaire en 1880, et à partir de 1912 on n'enseigna plus cette langue dans les écoles normales (Elenius 2006: 155; BOF 1921: 52). À l'instar de la situation française, les langues minoritaires persistent néanmoins en Suède, malgré une politique suédisante qui a longtemps dominé.

\subsection{La méthode directe}

Comme nous l'avons vu, les écoles primaires de Bretagne et de Tornédalie devinrent monolingues durant les années 1880. À la place d'un enseignement fondé sur la traduction se propagea une pratique de la méthode directe. En France, cette méthode fut l'objet d'un débat pendant les décennies suivantes, tandis qu'en Suède, la pratique avait précédé le débat. Alors que l'enseignement du suédois était occasionnel et facultatif dans les premières écoles tornédaliennes, l'enseignement exclusivement suédophone fut introduit à la fin des années 1880 , suite à l'initiative de l'évêque Lars Landgren et du pasteur P.O. Grape. (Elenius 2002)

En 1907, le chapitre cathédral du diocèse de Luleå chargea le professeur des langues finno-ougriennes à l'université d'Uppsala, Karl Bernhard Wiklund (1868-1934), d'élaborer des livres pédagogiques spécifiquement destinés aux élèves tornédaliens (Elenius 2006 : 157). La chaire de langues finno-ougriennes, instituée personnellement pour Wiklund en 1906, répondait au besoin d'expertise sur les questions touchant les régions du nord du pays (Karlsson 2000 : 18 ; Lundmark 2002; 67). Tout au long de sa carrière, Wiklund se consacra aux minorités du nord. Il fut chargé de plusieurs commissions pour l'État, dont la moindre ne fut pas de formuler des critères susceptibles d'identifier les différentes minorités dans les recensements en 1920 et 1930 (Karlsson $2000: 8,23)$. Son influence sur la politique des minorités suédoises est donc difficile à surestimer. À l'instar de Carré, Wiklund appliqua la méthode directe dans des manuels scolaires: en 1916, il publia un livre de l'élève et un manuel pour les institutrices.

Trois ans plus tard, suite à une motion parlementaire, une commission chargée d'examiner l'enseignement en Tornédalie fut formée. La commission publia ses résultats en 1921 dans le Rapport et proposition concernant l'école primaire dans les régions finnophones de la préfecture de Norrbotten (Betänkande och förslag rörande folkskoleväsendet $i$ de finsktalande delarna av Norrbottens län, BOF). Dans ce rapport, les membres de la commission décrivent les régions finnophones comme un problème, mais expriment également un sentiment d'optimisme pour l'avenir. Ils recommandent l'abandon total de la méthode de traduction dans l'enseignement. L'enseignement devrait désormais se faire exclusivement en suédois, mais sans que le finnois disparaisse de la région (BOF 1921 : 106). Pour aider l'enseignement, le rapport recommande les manuels écrits par Wiklund, systématisant la méthode directe. 


\section{L'hygiénisme}

\subsection{Un nouveau régime hygiénique}

16 Comme dans beaucoup d'autres pays européens, l'hygiène attira l'intérêt de nombreux hommes et femmes politiques et d'éducateurs en France et en Suède dans la deuxième moitié du XIX ${ }^{e}$ siècle (Björck 2008: 13, 17, 19). De plus en plus, l'importance et les techniques de la propreté firent l'objet d'articles, de livres et de règlements. En France, la création d'une école obligatoire, laïque et gratuite voulait aussi dire la création d'une école hygiénique. En 1882, des commissions d'hygiène scolaire furent formées pour surveiller l'hygiène des élèves et des locaux scolaires. L'historienne Julia Csergo parle d'un «véritable dressage hygiénique de la population » à cette époque. (Csergo 1988 : $120,110)$

17 Le philosophe finnois Olli Lagerspetz décrit l'intérêt croissant pour l'hygiène en termes non seulement quantitatifs, mais aussi comme changement qualitatif dans ce qu'il nomme régimes d'hygiène, thèse également soutenue par Csergo (op. cit., p. 2). Lagerspetz décrit un changement progressif allant d'une conception de la propreté basée sur l'ordre des choses vers une conception dans laquelle le nettoyage et l'eau jouent le rôle principal. Dans une société agrarienne où l'accès à l'eau était limité, la propreté fut maintenue au moyen de l'observance d'un certain ordre : le contact entre les choses et les sphères propres et sales fut minutieusement réglementé et contrôlé. Dans la société moderne, ce régime fut remplacé par un nettoyage fréquent des corps et des choses (Lagerspetz $2006: 17,139,142$ ). Tout en reconnaissant les avantages et les améliorations apportés par cet hygiénisme, dont par exemple l'élimination de certaines maladies épidémiques, nous pouvons nous intéresser aux implications culturelles et intellectuelles d'une nouvelle façon de penser le propre et le sale.

Le nouveau régime hygiénique se manifesta graduellement dans de nouvelles coutumes quotidiennes et habitudes sociales. Pourtant, celles-ci ne se produisirent pas spontanément. Elles furent plutôt le résultat de campagnes et de politiques mises en place pour enseigner à la population, surtout aux enfants, comment observer un comportement hygiénique correct (op. cit., pp. 139, 151). De plus, à cette époque, l'hygiène était une pratique qui s'inscrivait dans un projet républicain de modernité et de progrès scientifique, incarné dans le Dictionnaire de pédagogie et d'instruction primaire. Dans ce cadre, l'hygiène n'est point n'importe quelle propreté, « elle est au contraire une science et un art, très complexes, et où l'intervention du génie humain est à chaque instant nécessaire » (Pécaut $1887: 1301)$.

19 Suite à la nouvelle insistance sur la netteté, le malpropre prit dans une certaine mesure la place culturelle auparavant occupée par le pécheur : être sale était non seulement un signe de pauvreté, mais aussi, et plutôt, un signe de dépravation. Cette tendance se manifesta dans les bulletins pédagogiques français de l'époque :

[La malpropreté] est l'indice de la négligence, du désordre, de la paresse, du manque de goût, de la faiblesse de la volonté. Être propre est donc un devoir. L'esprit doit dominer la matière. - La propreté habituelle est l'indice des plus belles qualités morales. [...] [C]'est l'élégance du pauvre. (Ferroud $1897: 533$ )

20 En Suède, la tendance était la même. Dans les discours sociopolitiques de l'époque, les métaphores émaillant les récits des problèmes sociaux étaient souvent tirées des domaines médicaux et physiologiques. Les ingénieurs sociaux diagnostiquèrent la 
société en termes d'une dégénérescence qu'il fallait guérir en améliorant l'hygiène populaire (Björck 2008: 248). Vers le tournant du vingtième siècle, les communes prirent en charge l'infrastructure hygiénique, l'enlèvement des ordures, l'alimentation en eau et l'écoulement, auparavant laissés aux initiatives privées. Les autorités et les ingénieurs sociaux commencèrent à s'intéresser au comportement hygiénique de la population et aux solutions communales, surtout les bains publics. La malpropreté populaire devint un problème politique formulé en termes médico-hygiéniques. ( $O p$. cit., pp. 3, 7, 12)

21 L'hygiène attira aussi l'intérêt des éducateurs et fut enseignée dans le cadre de la connaissance du pays natal (Bäckström 2004: 38; Hellman 1922: 3) ${ }^{2}$. Un manuel de connaissance du pays natal incite les instituteurs et institutrices à parler de l'eau, du savon, de la propreté, du brossage des dents (faute de quoi on les perd, et on n'a rien avec quoi mâcher). Le texte parle aussi de la bonne façon de respirer hygiéniquement (non pas avec la cage thoracique, mais par le nez à l'exception de quand on parle ou quand on lit). (Sjöholm \& Goës 1916 : 56-57)

\subsection{La province crasseuse}

L'hygiénisme comportait aussi un intérêt anthropologique pour les questions de propreté et de saleté. Dans un essai sur les mœurs des Bretons publié en 1891, Carré s'étendra sur leur malpropreté et leur conception superstitieuse de la propreté avec tout le plaisir de l'exotisme du XIX siècle. Les Bretons portaient des vêtements et des coiffures antihygiéniques (certains enfants semblaient être "littéralement vêtus de trous »), leurs maisons étaient sombres et confinées, ils mangeaient mal et avec les mains. La culture bretonne était arriérée, en se promenant dans la région, on se trouvait parfois « en plein moyen âge ». (Carré 1891 : 469, 472, 477, 486)

On ne s'étonnera pas après cela que la propreté soit peu connue en Bretagne. On ne se lave guère que le dimanche. Les paysans mettent une sorte de point d'honneur à ce que leurs habits portent les traces de leur travail.

Sans ces traces, continue Carré, les Bretons craignent «qu'on ne les prenne pour des paresseux. La propreté leur semble un luxe qui n'est pas fait pour eux ». Toute cette négligence influence négativement la morale bretonne, car à «la malpropreté et à la misère s'allient très bien la mendicité et l'ivrognerie » (op. cit., p. 477). Enseigner le bon comportement hygiénique fut ainsi une affaire particulièrement urgente en Bretagne.

Dans les discours pédagogiques portant sur la Tornédalie, la crasse jouait un rôle similaire. L'amélioration des conditions hygiéniques fut une question importante dans le Rapport et proposition concernant l'école primaire dans les régions finnophones de la préfecture de Norrbotten : «L'hygiène générale à l'intérieur du pays de Norrbotten est de plusieurs points de vue moins satisfaisante » (BOF 1921 : 181). Dans son manuel destiné aux enfants parlant le meänkieli, Wiklund expliquait que l'éducation en «étiquette et hygiène " (hyfsning och hygien) était de la responsabilité de l'école. Tâche guère facile, assurait Wiklund puisque, çà et là, ces phénomènes "sont trop peu connus et peu pris en considération, oui, même considérés comme appartenant à une méprisable laïcité pécheresse ». (Wiklund 1916:21) 


\section{Propreté et langue maternelle}

\subsection{L'hygiénisme dans la méthode directe}

Selon la méthode directe, l'instituteur était appelé à montrer ou à mettre en scène ce qu'il voulait dire pour réussir à se faire comprendre sans se livrer à la traduction. Cette manière de démontrer ou d'illustrer était tirée de la méthode pédagogique nommée laleçon de choses. Cette méthode à son tour, fort influente dans la pédagogie de l'école Jules Ferry, s'appuyait sur la tradition allemande et suisse de l'Anschauungsunterricht, importée et francisée à l'époque de l'école républicaine sous le nom de méthode d'intuition ou méthode intuitive (Reinfried 1990: 127; Puren 2004:225)³. Même si Carré souligne l'importance de ne pas mélanger les leçons de langage avec les leçons de choses, l'influence de ces dernières au niveau de la structure des exercices est évidente dans sa méthode directe. L'idée principale qui consiste à fonder l'enseignement sur l'observation et l'étude spontanées des objets et actions concrets, fut fort présente dans la méthode directe carréenne. D'autres points en commun entre l'Anschauungsunterricht et la méthode Carré sont indiqués par Puren :

primauté accordée à l'oral à travers les exercices de langage, place centrale du dialogue enseignant-élève, importance de la répétition et de l'imitation, approche inductive de la grammaire, appel constant à l'activité physique des élèves, progressivité des contenus d'enseignement.

Puren rappelle aussi que "l'Anschauungsunterricht remplissait pour l'Allemagne la même fonction d'homogénéisation linguistique que celle exercée plus tard par la méthode Carré en Bretagne » (op. cit., p. 236). L'idée principale de la méthode était que dès l'entrée à l'école, les élèves allaient se trouver dans un milieu exclusivement francophone. La tâche du professeur était alors d'enseigner la langue nationale dans cette langue même. Afin d'éviter des traductions dans la langue maternelle des élèves, l'instituteur montrait des objets aux enfants et leur faisait faire des gestes tout en leur faisant répéter les mots correspondant à ces actes et ces objets. Souvent, les leçons parlaient des thèmes de la propreté et de la saleté, des procédés de nettoyage du corps et des choses.

Dans la méthode intuitive, les élèves étaient invités à observer les qualités des objets, à les comparer les uns avec les autres. Souvent, dans la méthode directe, il s'agissait d'exposer un élève exemplaire et de le contraster avec un élève présentant un comportement et une apparence non souhaitables :

Eugène est très désordonné, Il est toujours mal peigné, Très souvent il salit sa veste, Et c'est ainsi pour tout le reste. On le gronde, on le punit; mais Il ne se corrige jamais - sa sœur Marguerite, au contraire, Quoique plus jeune que son frère, (Elle a huit ans, il en a neuf), Est toujours propre comme un œuf. [...] C'est que Marguerite est très soigneuse, Raisonnable et travailleuse. (Carré 1889 : 64 ; majuscules dans le texte)

Dans cet exemple, les vertus de la petite Marguerite sont rendues visibles dans son apparence sous forme d'une propreté exemplaire. Parallèlement, les vices de son frère se manifestent sur son corps. Sa malpropreté en sont le marqueur extérieur.

Dans les manuels publiés en 1898, cette fois destinés non seulement aux provinces mais également à l'enseignement des élèves des colonies, Carré donne l'exemple d'Émile, prénom portant un certain héritage dans le discours pédagogique. Émile représente " un bon élève ", il se lève de bonne heure, il se lave le visage, le cou, les oreilles, il 
peigne ses cheveux. Émile est " toujours propre ", " soigneux » et, en écrivant, il ne se tache jamais les mains avec de l'encre. (Carré 1898 : 142)

Suivant les idées pédagogiques suisses-allemandes, les exercices carréens incitaient souvent les instituteurs à mettre en scène de petites représentations dans la salle de classe. Un élève se met en face des autres et effectue une sorte de chorégraphie détaillée par l'instituteur ou l'institutrice. L'exemple de la petite Henriette qui se lave les mains en est typique :

Henriette prend la cruche; elle la soulève; elle verse de l'eau dans la cuvette; elle repose la cruche à terre. Puis elle prend le savon, elle le met dans l'eau; elle frotte ses mains avec le savon [...]. Ses mains étaient sales tout à l'heure ; maintenant elles sont propres. (Carré 1889 : 81)

Dans le contexte pédagogique suédois, le concept d'Anschauung était connu sous le terme åskådning (observation, intuition). L'influence de la pédagogie allemande sur les manuels de Wiklund ne fait aucun doute; elle est même confirmée par le rapport parlementaire concernant les régions finnophones.

Au moyen d'observation et de travail, on éclaircit les concepts concrets sans emploi de traductions, à l'exception des cas d'urgence; l'activité propre des enfants est revendiquée d'une manière simple et maniable. (BOF 1921 : 110)

Comme dans la méthode Carré, l'hygiène est un thème important dans les exercices de Wiklund. Dans ses instructions aux institutrices, il souligne la place centrale dans l'enseignement des connaissances pratiques comme le nettoyage, la ventilation, le brossage des dents et l'importance de ne pas manger avec les mains ou de cracher par terre. Dans un exercice de suédois, les enfants apprennenttous les mots associés au lavage à la brosse. (Wiklund $1916: 46$ )

Puisque l'activité des élèves était centrale dans la théorie pédagogique à la base des méthodes de Wiklund et de Carré, les enfants étaient censés effectuer les actes dont parlaient les exercices dans les livres. Le principe d'enseignement était d'établir des liens forts entre les choses et les actes réels et les mots de la langue nationale. Souvent, le synopsis de la leçon était qu'un élève effectuait la chorégraphie décrite dans le manuel devant ses camarades, minutieusement dirigé par l'institutrice ou l'instituteur. Nous pouvons alors imaginer des salles de classe en Bretagne et en Tornédalie, où les jeunes élèves sont en train de regarder un camarade, fébrilement occupé à se laver ou à brosser le plancher, tout en répétant à haute voix «Lucien frotte ses mains avec le savon » ou « Anna nettoie la salle de classe ».

\subsection{La différence régionale}

Quel rôle jouait donc l'hygiénisme dans le discours de la méthode directe en Bretagne et en Tornédalie? En décrivant les Tornédaliens et les Bretons comme particulièrement malpropres, la différence régionale qu'ils représentaient était pour ainsi dire localisée sur la surface de leurs corps, et par conséquent possible à faire partir, à nettoyer. Les exercices d'hygiène de la méthode directe transforment les enfants provinciaux et crasseux en élèves propres de la république et de la nation.

Dans leurs manuels scolaires, Carré et Wiklund construisent des exercices pour enseigner simultanément la propreté et la langue nationale. À travers ces exercices, la procédure hygiénique est attachée à la langue nationale. En exerçant la chorégraphie du lavage, en se lavant les mains "en français» ou "en suédois", les enfants 
accomplissent un devoir national et confirment leur loyauté envers la patrie. L'exercice de langue basé sur les principes de l'Anschauung devient ainsi un composant du projet d'assimiler les enfants alloglottes à la nation. Dans la méthode directe, l'hygiénisme structure les exercices autour du corps et organise l'effort nationalisant dans des procédures de propreté.

L'accent mis sur la propreté corporelle des élèves résolvait ainsi une tension dans la conception de la différence dans l'État-nation. En Suède aussi bien qu'en France, prévalait une conception ambiguë de la nature de la différence des alloglottes. Dans les discours pédagogiques français, les Bretons étaient parfois décrits comme étant d'une autre race et donc difficiles à assimiler véritablement. Parfois le problème était la religion qui conservait une forte emprise en Bretagne, parfois la cause était le milieu dans lequel ils vivaient; pour d'autres, le problème était que les Bretons étaient arriérés et que la tâche de l'école était de les faire progresser jusqu'au niveau du reste de la population. (Puren 2004 : 254-256)

Pour Carré, la différence des Bretons était due à l'isolement relatif de la région, à son tour la raison pour laquelle ils étaient arriérés. Cet isolement avait aussi créé un caractère très spécifique, un caractère dont Carré parle parfois en termes de « race » :

On peut aller plus vite avec eux, en raison [...] de certaines qualités inhérentes à leur race : s'ils ne sont ni vifs ni ardents, ils sont assidus, tenaces et réfléchis. (Carré $1888: 227)$

La race bretonne était formée par le milieu dans lequel grandissaient et vivaient les Bretons, mais elle reproduisait et renforçait aussi à son tour ce milieu.

[Le Breton] est routinier et manque d'initiative : ce n'est point défaut de courage ou de cœur, mais sentiment profond de son ignorance. Il se défie des idées et des choses qui sont hors de sa portée ; il aime mieux suivre la tradition, marcher dans l'ornière qu'il connaît. Cette conscience qu'il a de son ignorance et de son impuissance fait aussi qu'il préfère laisser les choses en l'état: de là cette insouciance de tout progrès. (Carré 1891 : 487-488)

En outre, continue Carré, le Breton préfère vivre dans la pauvreté que se fatiguer en cultivant de nouvelles terres. L'entêtement et la robustesse qui le caractérisent sont à leur tour le résultat du manque d'idées nouvelles trouvant leur chemin en Bretagne.

Dans la description carréenne, les Bretons et leur milieu isolé constituent un cercle d'influence dans lequel ils se renforcent et se construisent mutuellement. L'aridité et le manque d'influences extérieures en Bretagne forment des habitants attachés aux coutumes et soupçonneux des nouveautés et des progrès. Leur mode de vie traditionnel contribue à conserver l'état peu fécond des terres et reproduit la rareté des relations avec l'extérieur.

Cette conception des Bretons s'appuyait sur la théorie néolamarckienne qui, plutôt que le darwinisme, prévalut en France dans les premières décennies de la Troisième République. Le néolamarckisme soulignait l'importance et la transmission des acquis plutôt qu'une sélection naturelle des individus (Rabinow 1989 : 126-127; Saada 2007 : 90-92). Évolutionisme plus politiquement praticable et compatible avec les idées éducatives de la Troisième République que le darwinisme, le néo-lamarckisme ouvrait la voie aux mesures réformistes. Il fallait rompre le cercle de la mauvaise influence réciproque du milieu et du caractère des Bretons. La tâche du système éducatif fut alors de détacher le Breton de l'influence de son milieu, de « le faire sortir de lui-même et de son pays, lui élargir les idées, le franciser en un mot ». (Carré 1888 : 234) 

les discussions pédagogiques. Dans le Rapport et proposition, les descriptions de la population exemplifient bien les ambiguïtés de la conception de la minorité finnophone. Les membres de la commission établissent d'abord que le concept de race est peu utile pour comprendre la région, puisque les populations suédoises et finnoises se sont mélangées depuis des générations. Dans le rapport, nous nous trouvons néanmoins dans un discours racial: malgré le fait que les habitants échappent aux efforts typologisants des membres de la commission, ceux-ci font état d'un mélange présupposant une "pureté » théorique possible. Dans au moins un passage, ils se réfèrent à ce mélange en termes d'un "mélange de races "; dans un autre, ils parlent d'un « élément finnois » dans la population. (BOF 1921 : 23-24) Norrbotten, la langue parlée par un individu ne correspond souvent pas à sa race ou à son type. La langue finnoise (meänkieli) domine certains villages où la population est « du type décidément germanique ». À propos de cette situation, les membres de la commission emploient le terme de "vraie langue maternelle " (egentligt modersmål) pour désigner la langue qui correspond à la race ou au type (op. cit., p. 89-90). La commission en conclut qu'aucun signe externe, comme les noms propres ou la langue parlée, ne devrait être retenu comme preuve certaine de l'origine finnoise d'un individu. Ils revendiquent néanmoins qu'il faut faire un effort dans les lettres officielles pour différencier entre les finnophones et les Finnois (op. cit., p. 25-27). Dans un autre passage, les membres de la commission prétendent que le mélange des races à Tornédalie crée quelque chose de neuf, au-delà des deux éléments. Le climat, l'isolement et les conditions particulièrement dures dans la région donnent une certaine mélancolie à la population qui est, en somme, arriérée, mais possède un grand potentiel de développement. (Op. cit., pp. 41, 47)

Plutôt que d'un néolamarckisme prononcé, le Rapport et proposition est marqué par une théorie raciale détectant un état de mélange dans la région. Les membres de la commission cultivent une conception de la différence, qui opère au niveau racial, dans laquelle l'influence climatique, géographique, sociale et interrégionale joue un rôle. L'état de diffusion d'une différence dénotée par le concept d'“élément " pose le problème de la nationalité autrement que s'il s'agissait des individus dans une population sinon homogène. Du «mélange des races » résulte que l'« élément finnois » n'est plus détectable au niveau individuel, mais plutôt diffusé dans la population suédoise de "type germanique ». La nationalité, la suédocité, devient ainsi une sorte de macrosujet, au- delà de l'ensemble des individus concernés.

\subsection{Une technique pour faire disparaître la différence régionale}

Comme nous l'avons vu, Carré décrivait la différence des Bretons en termes de malpropreté. Selon lui, la crasse était partout en Bretagne, elle était même socialement appréciée et liée à d'autres vices. La malpropreté devient de cette manière un marqueur de la régionalité, un signe de la différence sur la surface du corps. La crasse corporelle incarne tout ce qu'il faut faire partir pour franciser les Bretons. En faisant de la crasse le signe de la différence régionale, celle-ci est extériorisée et rendue maniable, enlevable. L'accent sur la propreté dans le discours pédagogique devient ainsi une manière pour la pédagogie de pénétrer dans le cercle néolamarckien du renforcement 
mutuel entre la race bretonne et son milieu. Au moyen de l'exercice hygiénique, l'instituteur peut nettoyer les élèves de la crasse bretonne, les libérer de la différence régionale et créer la table rase que la pédagogie républicaine présupposait comme point de départ.

L'exercice dans lequel Henriette se lave les mains d'après une chorégraphie minutieuse est destiné à enseigner aux petits Bretons un comportement ou des habitudes hygiéniques corrects, exprimés en mots français. Les mots sont gravés dans leur mémoire et la propreté est en même temps attachée à la langue nationale, l'hygiène nouée à la France. En décrivant la procédure de lavage en langue nationale, on lui attache en même temps la propreté. Les mots sont imprimés dans les esprits, et simultanément, la langue nationale est liée à la propreté. Après avoir été éduqués à l'école nationale, les enfants alloglottes seront aussi bien francophones ou suédophones que propres. Comme le note Julia Csergo, «davantage que l'histoire d'une pratique, l'histoire de la propreté est d'abord histoire politique ». (Csergo $1988: 288$ )

Dans cette interprétation de l'hygiénisme mis en pratique dans la méthode directe résonnent les théories de Michel Foucault sur les techniques de discipline, de dressage pour créer des "corps dociles ", forme de pouvoir croissant et affinée dès la fin du XVII ${ }^{\mathrm{e}}$ siècle :

Techniques minutieuses toujours, souvent infimes, mais qui ont leur importance: puisqu'elles définissent un certain mode d'investissement politique et détaillé du corps, une nouvelle «microphysique » du pouvoir; et puisqu'elles n'ont pas cessé, depuis le XVII ${ }^{e}$ siècle, de gagner des domaines de plus en plus larges, comme si elles tendaient à couvrir le corps social tout entier. (Foucault $1975: 163$ )

\subsection{Conclusion}

Les discours pédagogiques en Tornédalie et en Bretagne traitant la différence dans la nation ne sont point identiques. Ils sont marqués par des courants intellectuels différents, des évolutionnismes différents, soulignant des aspects différents de la nationalité. En Tornédalie, la notion de race jouait un rôle principal, tandis qu'en Bretagne l'interaction des notions d'hérédité et de milieu dans un cadre néolamarckien posait le problème de l'assimilation différemment. Pourtant, ce qu'ils ont en commun est une ambivalence dans la conception des minorités, une ambivalence rendue maniable par le cadre d'interprétation et les techniques offerts par l'hygiénisme.

« Formation en série » est le terme employé par Mona Ozouf pour décrire l'école de la Troisième République, une école mettant en scène la même nation, n'importe la province ou la langue locale (Ozouf 1996 : 7). Pourtant, le discours de l'école des régions alloglottes formait un cas particulier ; il développait son propre caractère. Comme nous avons essayé de le montrer dans ce texte, l'hygiénisme a contribué à ce caractère particulier. Il a offert une solution à la question de la différence régionale, et a marqué le modèle exemplaire de ce que devrait signifier être français ou suédois.

51 Le discours de la différence dans la nation est différent en Suède et en France, mais l'hygiénisme y joue un rôle similaire en rendant la différence régionale maniable et en l'incorporant dans l'enseignement de la langue nationale. L'hygiénisme fait converger les conceptions de la différence et les techniques pour nationaliser les minorités linguistiques dans les deux cas. Le transfert des idées pédagogiques en Europe, dans ce 
cas l'hygiénisme et l'Anschauung, est le facteur qui fait de la création des identifications avec les États-nations une affaire internationale.

\section{BIBLIOGRAPHIE}

(1921). Betänkande och förslag rörande folkskoleväsendet i de finsktalande delarna av Norrbottens län, utarbetat av utav statsrådet och chefen för Kungl. Ecklesiastikdepartementet den 6 juni 1919 tillkallade särskilda sakkunniga. Stockholm. [Rapport et proposition concernant l'école primaire dans les régions finnophones de la préfecture de Norrbotten] [BOF].

ANDERSON, Benedict (1983). Imagined communities: reflections on the origin and spread of nationalism. Londres : Verso.

BJÖRCK, Henrik (2008). Folkhemsbyggare. Stockholm : Atlantis.

BOUTAN, Pierre (1998). De l'enseignement des langues : Michel Bréal linguiste et pédagogue. Paris : Hatier.

BOUTAN, Pierre, MARTEL, Philippe \& ROQUES, Georges (éds). (2001). Enseigner la région. Actes du colloque international, IUFM Montpellier, 4-5 février 2000. Paris : L'Harmattan.

BRUBAKER, Rogers \& COOPER, Frederick (2005). « Identity », in Frederick Cooper (ed.), Colonialism in question: theory, knowledge, history. Berkeley : University of California Press, 59-90.

BUISSON, Ferdinand (éd.) (1887). Dictionnaire de pédagogie et d'instruction primaire. Paris : Hachette.

BÄCKSTRÖM, Mattias (2004). Skick och ordning i fostrans paviljonger: Norrlands kulturhistoriska museum och centralfängelset i Härnösand. Université de Göteborg [Mémoire de master inédit Département de l'histoire des idées].

CARRÉ, Irénée (1888). « De la manière d'enseigner les premiers éléments du français dans les écoles de la Basse-Bretagne », Revue Pédagogique, tome XII, 217-236.

- (1889). Méthode pratique de langage, de lecture, d'écriture, de calcul, etc, plus spécialement destinée aux élèves des provinces où l'on ne parle pas français, et qui arrivent en classe ne comprenant ni ne sachant parler la langue nationale. Livre de l'élève. Paris : Armand Colin.

- (1891). «La Basse-Bretagne : ses habitants ses mœurs, ses usages, ses écoles », in JOST (éd.), Annuaire de l'enseignement primaire. Paris : Armand Colin.

- (1898). Le Premier livre de lecture pour l'enseignement du français usuel aux indigènes de nos colonies, ainsi qu'aux élèves des contrées de la France qui arrivent en classe ne comprenant pas, ne sachant pas parler la langue nationale. Paris : Armand Colin.

CHANET, Jean-François (1996). L'École républicaine et les petites patries. Paris : Aubier.

CHERVEL, André (2006). Histoire de l'enseignement du français du XVII ${ }^{e}$ au XX ${ }^{e}$ siècle. Paris : Éditions Retz. 
CHRIST, Herbert \& COSTE, Daniel (éds). (1990) Contributions à l'histoire de l'enseignement du français. Actes de la section 3 du Romanistentag d'Aix-la-Chapelle du 27 au 29 septembre 1989. Documents pour l'histoire du français langue étrangère et seconde 6. Paris.

CSERGO, Julia (1988). Liberté, égalité, propreté. La morale de l'hygiène au XIXe siècle. Paris : Albin Michel.

DIGEON, Claude (1959). La crise allemande de la pensée française (1870-1914). Paris : Presses universitaires de France.

ELENIUS, Lars (2002).“Det förbjudna finska språket: Språkpolitik och kulturell revitalisering i Tornedalen”, Provins, 4.<http://tidskrift.nu/artikel.php?Id=2421>

- (2006). Nationalstat och minoritetspolitik : Samer och finskspråkiga minoriteter i ett jämförande perspektiv. Lund : Studentlitteratur.

FERROUD, Ch. (1897). « La morale par les maximes, les exemples, les morceaux choisis », L’Enseignement pratique, 46.

FOUCAULT, Michel (1975). Surveiller et punir. Paris : Gallimard.

GOSNELL, Jonathan K. (2002). The Politics of Frenchness in Algeria, 1930-1954. Rochester : University of Rochester Press.

HELLMAN, Theodor (1922). Hembygdsundervisningen i folkskolan: Ett försök till ämnets metodiska planläggning. Härnösand : Härnösands Boktryckeri.

JOST, M. (éd.) (1891). Annuaire de l'enseignement primaire. Paris : Armand Colin.

KARLSSON, Christer (2000). Vetenskap som politik : K. B. Wiklund, staten och samerna under 1900-talets första hälft. Umeå : Umeå universitet, Kulturens frontlinjer 25.

LAGERSPETZ, Olli (2006). Smuts: en bok om världen vårt hem. Stockholm : Symposion.

LUNDMARK, Lennart (2002). Lappen är ombytlig, ostadig och obekväm" : svenska statens samepolitik i rasismens tidevarv. Umeå : Norrlands universitetsförlag.

MELLBERG, Margaretha (1996). Pedagogen och det skrivna ordet : skrivkonst och folkskollärare i Sverige 1870-1920. Göteborg : Université de Göteborg, Département d'histoire.

OZOUF, Mona (1996). « Préface », in Jean-François Chanet, L'École républicaine et les petites patries. Paris : Aubier.

PÉCAUT, Élie (1887). « Hygiène », in Ferdinand Buisson (éd.), Dictionnaire de pédagogie et d'instruction primaire. Paris : Hachette, 1299-1308.

PUREN, Laurent (2003). « Pédagogie, idéologie et politique linguistique. L'exemple de la méthode Carré appliquée à la francisation de la Bretagne à la fin du XIX ${ }^{\mathrm{e}}$ siècle ", Glottopol : Revue de socio-linguistique en ligne, 1, Quelle politique linguistique pour quel État-nation ? <http:// www.univ-rouen.fr/dyalang/glottopol>

- (2004). L'école française face à l'enfant alloglotte : contribution à une étude des politiques linguistiques éducatives mises en œuvre à l'égard des minorités linguistiques scolarisées dans le système éducatif français du XIX ${ }^{\mathrm{e}}$ siècle à nos jours. Université de la Sorbonne nouvelle [Thèse de doctorat inédite - UFR de didactique du français langue étrangère].

RABINOW, Paul (1989). French Modern: norms and forms of the social environment. Cambridge, Mass. : MIT Press.

REINFRIED, Marcus (1990). «Les origines de la méthode directe en Allemagne », in Herbert Christ \& Daniel Coste (éds), Contributions à l'histoire de l'enseignement du français. Actes de la section 
3 du Romanistentag d'Aix-la-Chapelle du 27 au 29 septembre 1989, Documents pour l'histoire du français langue étrangère ou seconde, 6. Paris. 126-156.

SAADA, Emmanuelle (2007). Les enfants de la colonie. Les métis de l'Empire français entre sujétion et citoyenneté. Paris : Éditions La Découverte.

SJÖHOLM, Lorentz Gottfrid \& GOËS, A. (1916). Handledning vid undervisningen i hembygdskunskap. I. Första skolåret. Stockholm : P. A. Norstedt \& Söner Förlag.

THIESSE, Anne-Marie (1999). « Democracy softens forces of change: Inventing national identity ». Traduit par Barry Smerin. <http://www.hartford-hwp.com/archives/60/062.html>

- (2001a). La création des identités nationales. Europe XVIII ${ }^{\mathrm{e}}-\mathrm{XX}{ }^{\mathrm{e}}$ siècle. Paris : Editions du Seuil.

- (2001b). «Par la région, pour la nation : Pratiques scolaires de la Troisième République », in Pierre Boutan, Philippe Martel \& Georges Roques (éds), Enseigner la région. Actes du colloque international, IUFM Montpellier, 4-5 février 2000. Paris : L'Harmattan.

WEBER, Eugen (1976). Peasants into Frenchmen: the modernization of rural France, 1870-1914. Stanford : Stanford University Press.

WIKLUND, Karl Bernhard (1916). Handledning vid den första undervisningen i svenska i skolorna i Tornedalen. Uppsala, Stockholm : Almqvist \& Wiksell.

\section{NOTES}

1. Nous employons ici le terme d'identification, plutôt que celui d'identité, suivant la critique de Brubaker et Cooper. Le concept d'identification a la vertu de distinguer entre l'expérience vécue dans la vie quotidienne et une politique visant à créer une communauté. Nous entendons par ce terme le but visé par les pédagogues, et non pas le résultat dans le sens d'un sentiment d'affiliation éventuel chez les élèves.

2. Hembygdskunskap, approximativement connaissance du pays natal, fut le nouveau nom de l'enseignement intuitif (åskådningsundervisning) dans le programme d'enseignement de 1919. À l'instar de la leçon de choses en France, le but de l'enseignement en connaissance du pays natal était de développer la faculté d'observation chez les enfants en examinant les alentours de l'école.

3. Reinfried retrouve les origines de la méthode directe en Allemagne au milieu du XVIII ${ }^{\mathrm{e}}$ siècle.

\section{RÉSUMÉS}

À partir des années 1880, la méthode directe (MD) fut employée pour enseigner la langue nationale aux élèves de l'école primaire dans les régions alloglottes des deux pays, la France et la Suède. La thématique de l'hygiène, fort présente dans les manuels de la MD, attachait les mots de la langue nationale aux procédures hygiéniques. La langue nationale était ainsi associée à la notion de propreté. L'hygiénisme présentant aussi un intérêt anthropologique pour les questions de propreté et de saleté, les Tornédaliens et les Bretons étaient décrits comme particulièrement malpropres. Leur malpropreté devenait alors un marqueur de régionalité sur la surface de leurs corps. Les exercices d'hygiène de la MD visaient à une transformation des enfants provinciaux et 
crasseux en élèves propres de la république et de la nation. Le discours de la différence régionale dans la nation était différent en Suède et en France, mais l'hygiénisme jouait pourtant un rôle similaire dans les deux pays, en rendant la différence régionale maniable dans le cadre de l'enseignement de la langue nationale.

Starting in the 1880s, the direct method (DM) was used in national language teaching in linguistic minority regions in Sweden and France. The focus on hygienic themes in pedagogical textbooks systematizing the DM attached the words of the national language to hygienic procedures. In this way, the national language was associated with a notion of cleanliness. Hygienism also entailed an anthropological interest in questions of cleanliness and dirtiness. The inhabitants of Brittany and Torne Valley were often described as particularly dirty, and this dirtiness became a sign of regionalism on their bodies. The DM exercises aimed at a transformation of the perceived dirty children of the province into clean pupils of the republic and nation. The discourses on regional difference within the nation differing between the two countries, hygienism yet played a similar role in Sweden and France, in making difference manageable in the framework of national language teaching

\section{INDEX}

Mots-clés : France, Bretagne, Suède, Tornédalie, langues régionales, langues nationales, régions alloglottes, minorités linguistiques, hygiénisme, méthode directe

Keywords : France, Brittany, Sweden, Torne Valley, regional languages, national Languages, linguistic minority regions, language minorities, hygienism, direct method

\section{AUTEUR}

JULIA NORDBLAD

Université de Göteborg, Suède

julia.nordblad@lir.gu.se 